\title{
Estimates of Breast Cancer Growth Rate and Sojourn Time from Screening Database Information
}

\author{
James Michaelson, PhD, ${ }^{*}$ Sameer Satija, AB,† Richard Moore, AB, $\ddagger$ Griffin Weber, BS, $\S$ Elkan Halpern," \\ Andrew Garland, 9 and Daniel B. Kopans, MD\#
}

A new method has been developed that can be used to estimate tumor growth rate from information on the numbers and sizes of breast cancers found at screening. With use of this method and information available for the tumors seen over the last decade at the Breast Imaging Division at the Massachusetts General Hospital (MGH), it appears that the median doubling time for invasive breast cancer is approximately 130 days. From this doubling time value, together with information on the sizes at which breast cancers become detectable on clinical grounds and by screening, it appears that the mean and median sojourn times for invasive breast cancers in the MGH population are approximately 1.7 and 1.3 years, respectively. [Key words: breast cancer, doubling time, growth rate, sojourn time] Journal of Women's Imaging 2003:5:11-19

A number of studies have estimated the growth rate of breast cancer from patients in whom the size of the tumor could be measured on two different occasions, often when the tumor could be seen on a review of an earlier mammogram, ${ }^{1-8}$ yielding estimates of the breast cancer doubling time ranging from 80 days to 260 days. $^{3-8}$ A number of other studies have estimated the breast cancer sojourn time, which is the time that it takes for tumors to grow from mamographically detectable size to clinically detectable size,${ }^{9-17}$ yielding values ranging from 1.3 to 4.2 years. ${ }^{9-17}$ We have developed a new method that can be used to estimate the tumor growth rate from information on the number of breast cancers found at screening and have applied this to the

\footnotetext{
*Assistant Professor of Pathology, Division of Surgical Oncology, Massachusetts General Hospital, Boston, MA; †Medical Student, Emory Medical School, Atlanta, GA; $\ddagger$ Director of Breast Imaging Research, Department of Radiology, Massachusetts General Hospital, Boston, MA; $\$$ Medical Student, Harvard Medical School, Boston, MA; "Statistician and Department of Radiology, Massachusetts General Hospital, Boston, MA; IIConsultant, Lexington, MA; \#Professor of Radiology, Department of Radiology, Massachusetts General Hospital, Boston, MA; **Assistant Professor of Surgery, Division of Surgical Oncology, Massachusetts General Hospital, Boston, MA.

The authors have disclosed that they have no significant relationships with or financial interest in any commercial companies pertaining to this educational activity.

Acknowledgment date: September 4, 2001.

Revision date: November 21, 2002.

Acceptance date: November 21, 2002.
}

data on the cancers seen at the Massachusetts General Hospital (MGH) Breast Imaging Division ${ }^{18}$ to provide estimates of the intrinsic breast cancer growth rate and sojourn time in our patient population.

\section{MATERIALS AND METHODS}

\section{Data Set and Basic Definitions.}

Features of the 810 invasive breast cancers seen at the MGH Breast Imaging Division between 1990 and $1999^{18}$ were reviewed. Carcinomas in situ were not included. Invasive breast cancers were divided into categories: first-screen-detected cancer (invasive breast cancer identified by mammography in an asymptomatic woman at her first screen at MGH), subsequent-screen-detected cancer (invasive breast cancer identified by mammography in an asymptomatic woman who has had at least one previous negative screening mammogram result at $\mathrm{MGH}$ ), intervening cancer (invasive breast cancer identified by means other than a screening mammogram in a woman who has had a least one previous negative screening mammogram result at MGH), and never-screened cancer (invasive breast cancer identified by means other than a screening mammogram in a woman who has no history of mammography at $\mathrm{MGH})$. Note that we have adopted the term "intervening cancer" to distinguish it from the term "interval cancer," which is usually used to describe a tumor arising after negative examination results but within a specified period of time. For the cancers that arose in women who had had a previous negative mammogram result (intervening and subsequent-screendetected cancers), it was possible to determine the length of time from the previous negative examination result until the time when the cancer was diagnosed. We used the pathologic measure of the largest tumor diameter as the size of each cancer. As described elsewhere in this issue of the Journal of Women's Imaging, ${ }^{19}$ the median size of the never-screened cancers at $\mathrm{MGH}$, and therefore the median size at which breast cancers become detectable by means other than screening (the median $\mathrm{S}_{\mathrm{p}}$ ), is $15 \mathrm{~mm}$, and the distribution of values around this mean can be seen from data on the full distribution of the sizes of these never-screened cancers. Similarly, from data on the sizes of the screen-detected cancers ${ }^{18}$ (firstscreen-detected and subsequent-screen-detected cancers), it was possible to estimate the median value (the median $S_{m}$ ), which is $7.5 \mathrm{~mm}$, together with the distribution of values around this median value, for the size at which breast cancers become detectable by screening. ${ }^{19}$ The screening yield is defined as the ratio of invasive breast cancers found to the number of screening mammograms obtained. The cancers found 
on the first mammogram are often referred to as the prevalent cancers, whereas cancers found on subsequent mammograms are often referred to as the incident cancers. Calculations of the screening yield for both types of cancers were generated and values for the incident cancers were sorted by the amount of time since the previous negative mammogram result.

\section{RESULTS}

For reasons that can be seen in Figure 1, the faster the tumor growth rate, the greater the abundance of cancers seen at screening. In addition, by expressing this feature in algebraic form (see Appendix), it is possible to make an estimate of the median tumor doubling time (equation 14, Appendix). When used with information on the abundance of tumors between $6 \mathrm{~mm}$ and $41 \mathrm{~mm}$ seen at screening at the MGH Breast Imaging Division (firstscreen-detected cancers and subsequent-screen-detected cancers), ${ }^{18}$ equation 14 generated an estimate of the median doubling time of approximately 130 days (Figure 2). A similar estimate made from just those cancers found on the first screening examination (first-screendetected cancers) yielded a doubling time estimate of 132 days. No significant age-specific differences in growth rate were found with equation 14 when subgroups of women, sorted by age, were analyzed. Calculations of information on the abundance of tumors smaller than $6 \mathrm{~mm}$ (of which there were 50 in our data set) yielded somewhat shorter estimates of the median doubling time (36-94 days; Figure 2). This could reflect either experimental error (because there were relatively few tumors in our data set smaller than $6 \mathrm{~mm}$ ) or the possibility that tumors smaller than $6 \mathrm{~mm}$ might have a somewhat faster growth rate than larger tumors, a feature long recognized in breast cancer. ${ }^{2,20,21}$

Another expression of the breast cancer growth rate is the sojourn time. The sojourn time is the time that it takes the cancer to grow from a size at which it becomes detectable by screening $\left(\mathrm{S}_{\mathrm{m}}\right)$ to a size detectable clinically $\left(S_{p}\right)$ (equation 4, Appendix). As reported elsewhere in this issue of the Journal of Women's Imaging, ${ }^{19}$ the median $\mathrm{S}_{\mathrm{p}}$ value at $\mathrm{MGH}$ is approximately $15 \mathrm{~mm}$, whereas the median value of $S_{m}$ is approximately 7.5 $\mathrm{mm}$. To grow from $7.5 \mathrm{~mm}$ to $15 \mathrm{~mm}$ requires approximately 3.7 tumor volume doubling times. It follows that the median sojourn time for the MGH population should be approximately 16 months (130 days $\times 3.7$ or approximately 1.3 years). Based on the 130 -day tumor doubling time value and a determination of the variation in doubling times that comprise the range of sojourn times (Figure 3), it follows that the mean sojourn time is approximately 20 months (approximately 1.7 years) (Figure 4). Approximately 65\% of cancers should have sojourn times of between two and eight doubling times (sojourn time $=0.7-2.1$ years), whereas only $10 \%$ of cancers would appear likely to have sojourn times of
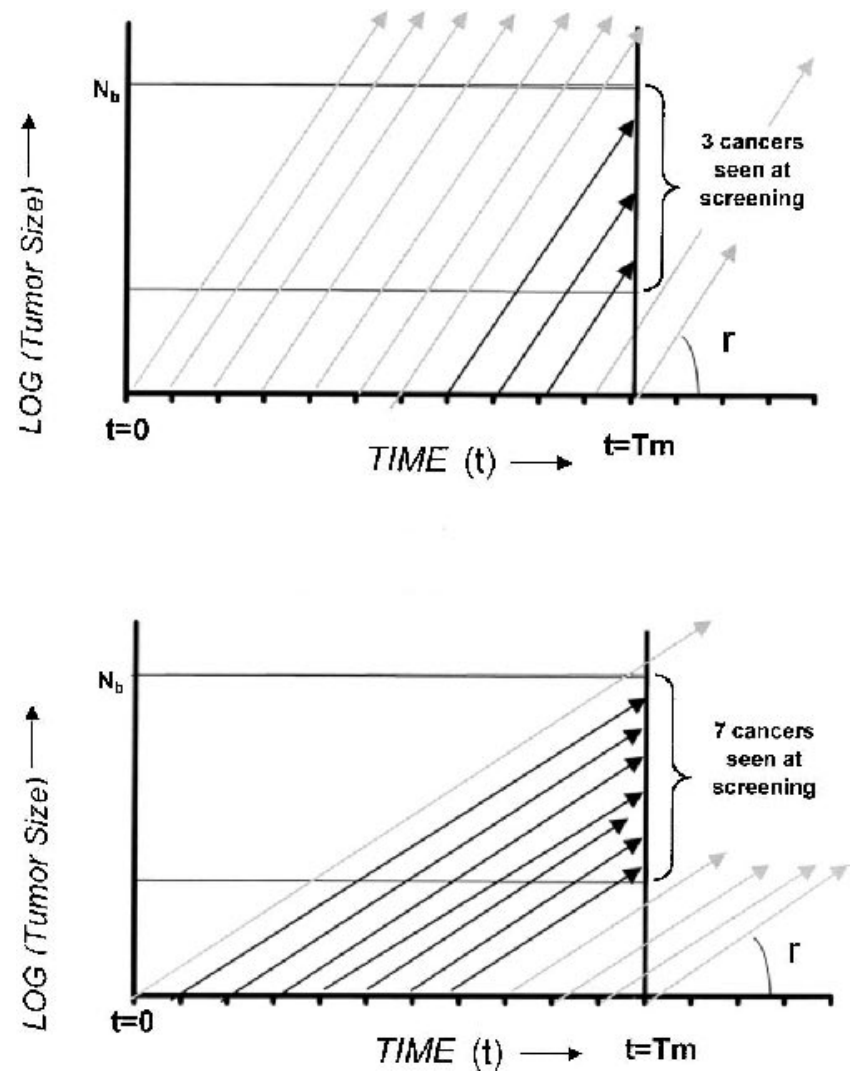

Figure 1. Diagram illustrating the relationship between the number of breast cancers seen at screening and the tumor growth rate. Here, the natural logarithm of tumor size, in number of cells, $\mathrm{N}$, is shown in the $\mathrm{Y}$ axis, and time $(\mathrm{t})$ is on the $\mathrm{X}$ axis. Therefore, tumor growth appears as a straight line, with a slope, $r$, where $r=\ln (2)$ / doubling time and where the growth equation is $\mathrm{N}=\mathrm{N}_{0} e^{\text {rt }}$ where $\mathrm{N}_{0}$ is the number of cells at time $\mathrm{t}=0$ and $e$ is the exponential constant. The two figures shown here are identical in terms of breast cancer incidence, with 11 cancers shown arising between time $\mathrm{t}=0$ and time $\mathrm{t}=\mathrm{t}_{\mathrm{m}}$, at which time mammographic screening is carried out. However, tumor growth rate, $r$, is faster (and therefore the growth line is steeper) in the bottom figure than in the top figure. As a result, more cancers between sizes $N_{b}$ and $N_{s}$ are seen at mammography for the slower (bottom) growth rate than the faster (top) growth rate. Tumors found at screening are indicated in black, whereas tumors either too small to be found at screening, or which have reached a clinically detectable size before screening, and therefore are not seen at the time of the screening examination, indicated in gray.

longer than eight doubling times, thereby being longer than 2.8 years (Figure 3 ). For approximately $15 \%$ of cancers, $S_{m}$ is greater than $S_{p}$; these cancers would be expected to always be detected by nonmammographic methods before they could be detected by mammography and, in fact, a small number of tumors seen at the MGH Breast Imaging Division fulfill this expectation. ${ }^{18}$ Of course, these estimates of sojourn time are what would be expected for an intrinsic tumor doubling time of 130 days, but this 130-day value, made with equation 14 (Figure 2), defines a measure of the median tumor doubling time and does not provide any information on the degree of tumor-to-tumor variation in doubling 


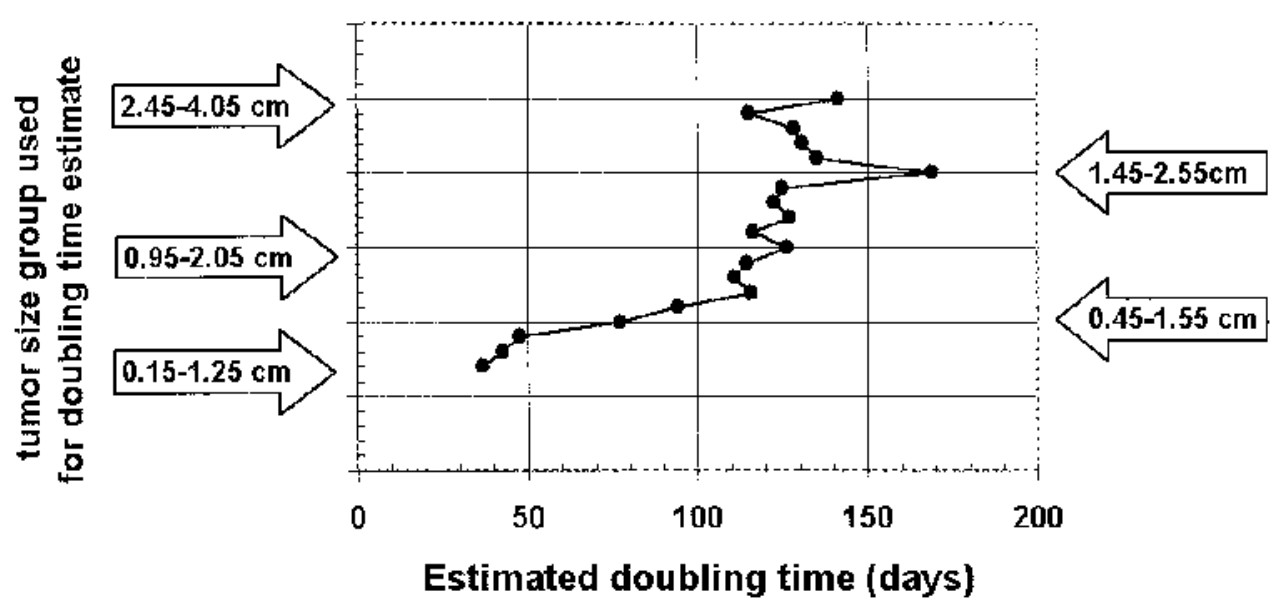

Figure 2. Estimates of the median invasive breast cancer doubling time. The solution was determined with use of equation 14 and executed on Excel (Microsoft, Redmond, WA) from data on the tumors seen at screening and as palpable masses. ${ }^{18}$ Each group contains tumors spanning a size range of $11 \mathrm{~mm}$ and each group shown differs by 1-mm increments.

time. It was possible to estimate the effect that different degrees of variation in the intrinsic rate of tumor doubling might have on the woman-to-woman variation in sojourn time, revealing that that the variation in $S_{m}$ and $S_{p}$ probably contributes far more to the woman-towoman variation in sojourn time than any likely degree of tumor-to-tumor variation in the intrinsic growth rate of breast cancer (Figure 4).

We analyzed the tumors found after a negative mammogram result (intervening and subsequent-screendetected cancers), because it has long been appreciated that, during a period of time reflecting the sojourn time, these tumors will be reduced in size and abundance (Figure 5). ${ }^{9-17,20}$ For the highly idealized case in which there is but a single sojourn time, this period of time will correspond to the sojourn time (Figure 5); using the estimates of the range in sojourn times described herein, it can be expected that meaningful reductions in the sizes and abundance of tumors seen at screening should occur only for screening intervals of less than 1 year (Figure 6). This is essentially borne out by the results from the MGH data set. For example, we found that the sizes of the intervening cancers tended to be reduced, but only if found within approximately 9 months of a negative screening examination result (Table 1, Figure 7). The sizes of the subsequent-screen-detected cancers also were reduced if found within approximately 9 months of a negative screening examination result (Table 2). We also found that the abundance of the intervening cancers was also markedly reduced during the 9 months after a negative mammogram result (Figure 8). Similarly, the abundance of the subsequentscreen-detected cancers (the screening yield) was markedly reduced during the 14 months after a negative mammogram result (Figure 9), after which time it proved to be remarkably close to the value of approximately four prevalent cancers per 1,000 mammograms seen for first-screen-detected cancers (Figure 9).

\section{DISCUSSION}

A number of studies have made estimates of the breast cancer doubling time from tumor sizes observed at two points in time, usually by reexamining previous mammograms for an earlier sign of the cancer (Table 3). Using this approach, Fournier et $\mathrm{al}^{3}$ found breast can-
Figure 3. Cumulative distribution and distribution of the various number of doublings that should be expected in the breast cancer sojourn times present within the population of women as a whole, based on variation in the values of $\mathrm{S}_{\mathrm{m}}$ and $\mathrm{S}_{\mathrm{p}}{ }^{18}$

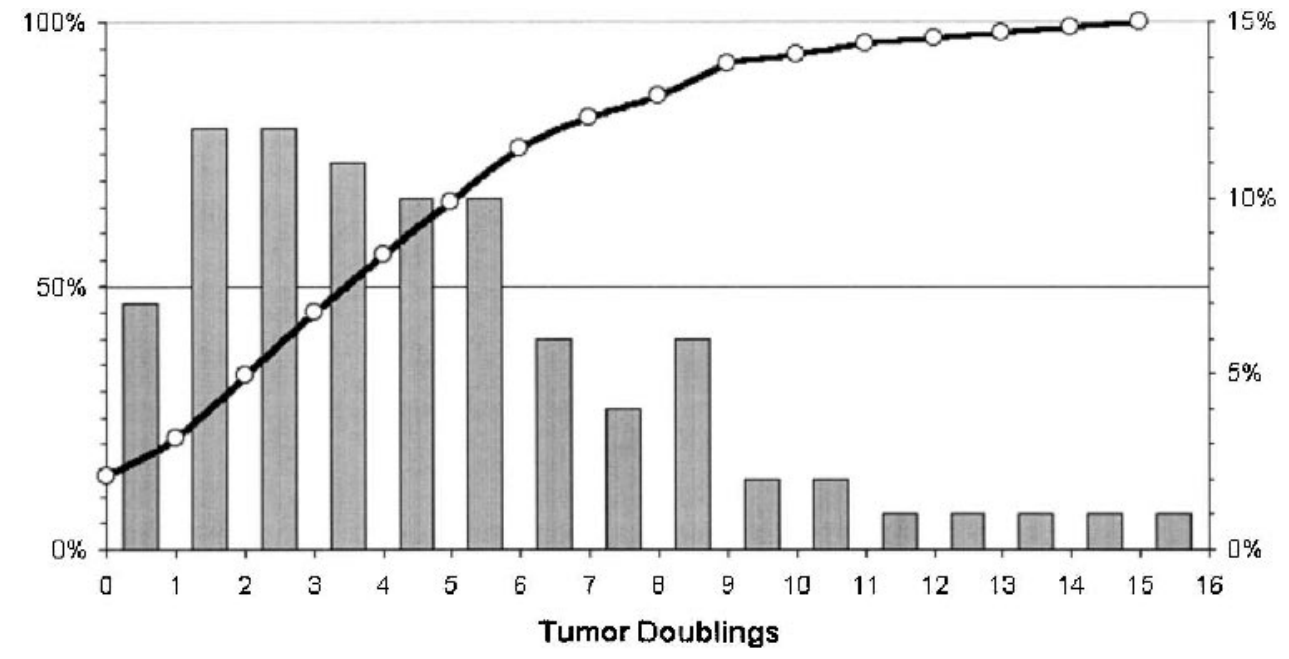




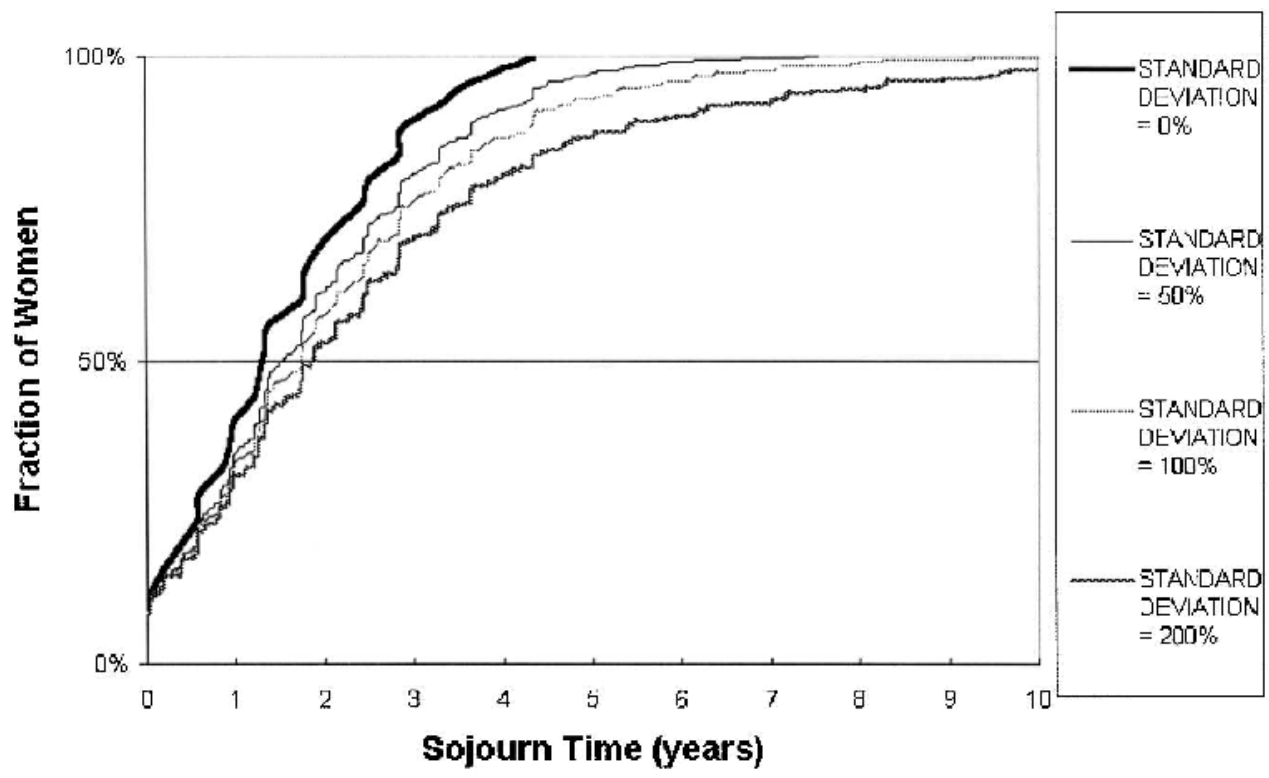

Figure 4. Cumulative distribution of breast cancer sojourn times based on estimates of the variation in $S_{m}$ and $S_{p}{ }^{18}$ and on an estimated median tumor doubling time of 130 days. The degree of variation around the median doubling time is unknown, and therefore the effects of various standard deviations (expressed as a percentage of the median value of 130 days) on the cumulative distribution of the breast cancer sojourn times are also shown.

cers to have a geometric mean doubling time of 225 days, whereas Lundgren ${ }^{4}$ found the mean doubling time to be 211 days, Spratt et $\mathrm{al}^{5}$ found the median doubling time to be 260 days, and Kuroishi et $\mathrm{al}^{7}$ found a geometric mean doubling time of 174 days. Peer et al, ${ }^{8}$ who sorted patients by age, found the geometric mean dou-

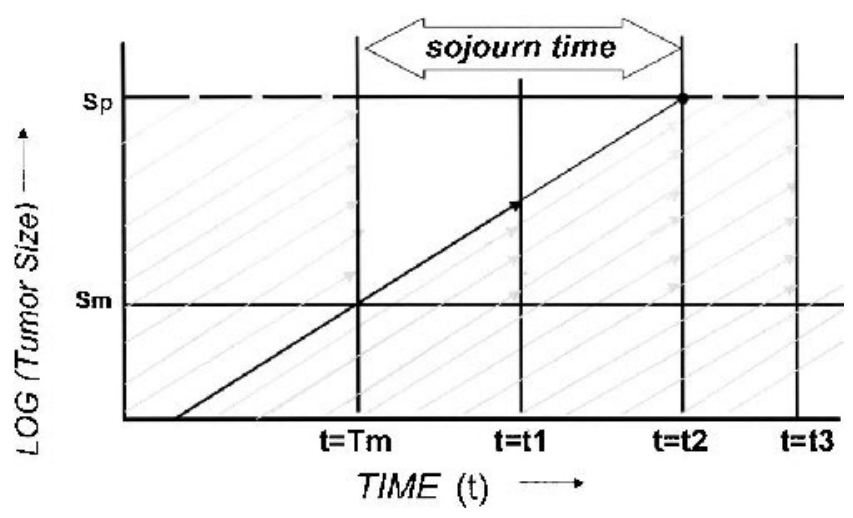

Figure 5. Diagram illustrating the effect of a previous negative mammogram result (at time $t=T_{m}$ ) on the abundance and size of tumors seen at various times after that mammogram (t1, t2, t3). Shown is the case for a single sojourn time (the time that it takes for a tumor to grow from size $S_{m}$ to size $S_{p}$, where $S_{m}$ is the size at which tumors will become detectable by mammography and $S_{p}$ is the size at which tumors will become detectable by palpation), and that $t_{2}-T_{m}=$ the sojourn time. Tumors found at screening are indicated by arrows ending in points whereas tumors found as palpable masses are indicated by arrows ending in closed circles. Note that tumors will be reduced in size and abundance if found within a period of time less than the sojourn time (i.e., for times $\mathrm{t}<\mathrm{t} 2$ ). Therefore, this diagram illustrates that, if screening is carried out at time t1, which occurs before the sojourn time, the number and sizes of any tumors seen at screening will be reduced. However, this protective effect will last only as long as the sojourn time so, for example, the number and distribution of sizes seen at times $\mathrm{t} 2$ and $\mathrm{t} 3$, both of which occur after the sojourn time, are equivalent. bling time of breast cancer to be 80 days for women younger than 50 years of age, 157 days for women aged 50-70 years, and 188 days for women older than age 70. One of the difficulties of such measurement is the inherent inaccuracy of estimating the actual tumor size from the mammographic image. We have found that the tumor sizes seen on the mammogram show little correlation with the tumor size measured at the time of pathologic analysis (Michaelson and Weber, unpublished data). Herein we have presented a new method for estimating the median breast cancer doubling time that relies on the number of tumors seen at screening and tumor sizes assessed on pathologic analysis. With use of this new method and data on the sizes of the tumors found at screening over the past decade at the MGH Breast Imaging Division, ${ }^{18}$ we have found that the median breast cancer doubling time is approximately 130 days. Reassuringly, this value is roughly in accord with the estimates of the breast cancer growth rate found in a number of previous studies relying on serial mammographic measurements (Table 3). ${ }^{3-7}$

With the information on the intrinsic rate of breast cancer growth (median doubling time of approximately 130 days), together with the estimates of the sizes at which individual invasive breast cancers will become detectable by mammography and the sizes at which individual invasive breast cancers will become detectable in the absence of mammography, ${ }^{18}$ we have been able to determine that the median and sojourn times are approximately 1.3 and 1.7 years, respectively. Of course, the sojourn time values reported herein are probably specific for the population at MGH and will be different in other populations because of differences in cancer detection. Indeed, a number of other studies have made estimates of sojourn times, ranging from 1.3 years to 4.2 years $^{9-17}$ (Table 4). As we have noted previously, ${ }^{18}$ the median size of tumors in unscreened women seen 
Figure 6. Reductions in the screening yield and median size of the screen-detected tumors expected to be found at screening carried out at various intervals, based on a tumor doubling time of 130 days, and the distribution of values of $S_{m}$ determined from the screening database. ${ }^{18}$

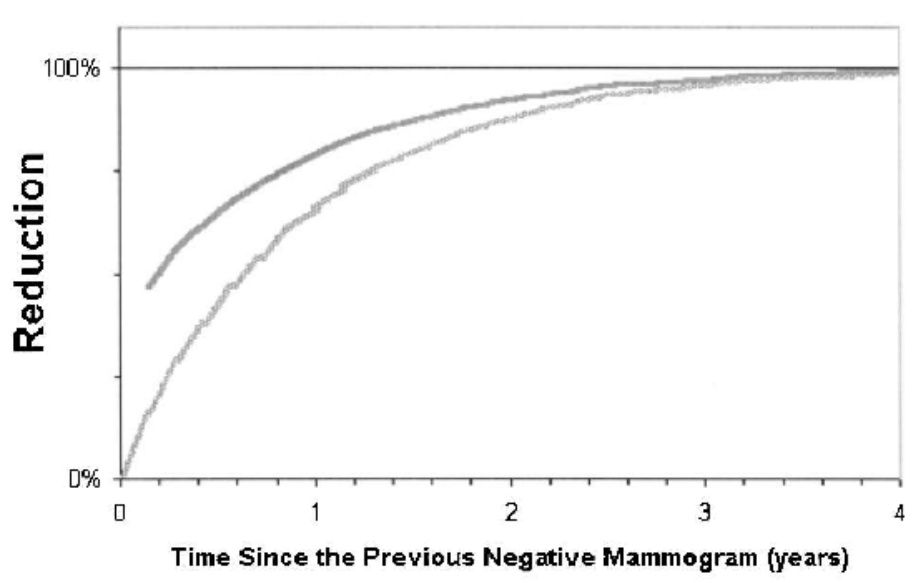

at MGH during the past decade was approximately 15 $\mathrm{mm}$, whereas the median size of tumors found by Tabar and colleagues ${ }^{21}$ in unscreened women reported by from 1977 to 1985 was approximately 20 $\mathrm{mm}$ and the median size of cancers found by Tubiana and colleagues ${ }^{22,23}$ in the premammographic years of 1954-1979 was approximately $40 \mathrm{~mm}$. Because tu-

Table 1. Sizes of Intervening Cancers Since the Previous Negative Mammogram

\begin{tabular}{lcccc}
\hline $\begin{array}{l}\text { Time Since } \\
\begin{array}{l}\text { The Previous Negative } \\
\text { Mammogram (y) }\end{array}\end{array}$ & $\mathrm{n}$ & $\begin{array}{l}\text { Mean } \\
\text { Diameter } \\
\text { (cm) }\end{array}$ & $\begin{array}{l}\text { Median } \\
\text { Diameter } \\
\text { (cm) }\end{array}$ & t-test \\
\hline $0.095-0.62$ & 30 & 1.42 & 1.35 & $\mathrm{p}<0.03$ \\
$0.42-0.72$ & 30 & 1.59 & 1.4 & $\mathrm{Ns}$ \\
$0.65-0.87$ & 30 & 1.72 & 1.45 & $\mathrm{Ns}$ \\
$0.73-1.06$ & 30 & 1.68 & 1.5 & $\mathrm{Ns}$ \\
$0.88-1.21$ & 30 & 1.55 & 1.4 & $\mathrm{Ns}$ \\
$1.06-1.52$ & 30 & 1.51 & 1.4 & $\mathrm{Ns}$ \\
$1.3-1.8$ & 30 & 1.94 & 1.5 & $\mathrm{Ns}$ \\
$1.52-2.33$ & 30 & 2.25 & 1.55 & $\mathrm{Ns}$ \\
$1.84-3.1$ & 30 & 1.95 & 1.5 & $\mathrm{Ns}$ \\
$2.46-5.15$ & 30 & 1.60 & 1.5 & $\mathrm{Ns}$ \\
$3.31-13.1$ & 27 & 1.53 & 1.5 & - \\
$0.095-13.1$ & 179 & 1.68 & 1.5 & \\
$0.42-5.15$ & 117 & 1.70 & 1.5 & \\
\hline
\end{tabular}

The 179 intervening cancers were ordered from shortest to longest time since the previous negative mammogram and all possible sequential groupings of 20 to 40 tumors were examined by Excel spreadsheet analysis. This yielded approximately 3,000 groups. A number of representative groups $(n=30)$ are shown. Of these approximately 3,000 groups, 131 groups of tumors, those containing tumors found within 0.7125 years of the previous negative exam, were significantly $(p<0.05)$ smaller than the mean size of the tumors found after this time; for no group containing tumors found after 0.7125 years was there any significant reduction in mean tumor size, in comparison to the tumors found later (Figure 7). Of these 131 groups, the one with the highest degree of statistical confidence $(p=0.000204)$ was the group that contained 22 cancers found between 0.548 and 0.660 years after the previous negative exam; these 22 tumors were reduced in diameter by approximately $40 \%$, with a mean size of $10.4 \mathrm{~mm}$, in contrast to the mean size of $16.8 \mathrm{~mm}$ for the group of 179 intervening tumors as a whole, and $17.7 \mathrm{~mm}$ for the group of 145 intervening tumors found more than 0.660 years after the previous negative exam. The mean size of all of the tumors in the 131 groups of tumors that were significantly smaller because they were found within 0.7125 years the previous negative exam were reduced in diameter by approximately $30 \%$, with a mean size of $13.0 \mathrm{~mm}$, in comparison to the mean size of $16.8 \mathrm{~mm}$ for the group of 179 intervening tumors as a whole. mors that are clinically detected are found at smaller sizes, the sojourn time gets shorter, and, in fact, Chen et al, ${ }^{16,17}$ with use of a Markov model, made estimates from the studies of Tabar and colleagues, ${ }^{21}$ yielding estimates of mean sojourn times of 2.4 years for women aged 40-49 years, 3.7 years for women aged 50-59 years, and 4.2 years for women aged 60-69 years.

The intrinsic rate of tumor growth that we have estimated herein describes the rate of growth of tumors in their invasive stage only and does not apply to the long periods of hyperplasia and carcinoma in situ that may precede invasive breast cancer. ${ }^{24}$ The method presented herein also provides an estimate of the median doubling time but does not provide information on the degree of tumor-to-tumor variation in doubling time. We suspect that it may be possible to generate estimates of the rates of growth during the early preinvasive period of tumor development and to make estimates of the degree of tumor-to-tumor variation in doubling time of invasive breast cancers, and these will certainly be areas for further analysis.

One benefit of increasing the frequency of screening is that the tumors seen at mammography should be reduced in size. ${ }^{25,26}$ However, we found this to occur for only those cancers found within approximately 1 year of the previous examination. Because very few women are now screened more often than once per year, this benefit is not achieved, and this alone raises the questions of whether screening more frequently than once a year may have some benefit. $1,25,27,28,29$

\section{APPENDIX}

The following is the method for estimating the rate of growth of invasive breast cancer from the abundance of cancers seen at screening.

Over the rather narrow ranges of sizes at which most tumors are seen (approximately 1-2 cm), it appears likely that invasive breast cancer growth is exponen- 


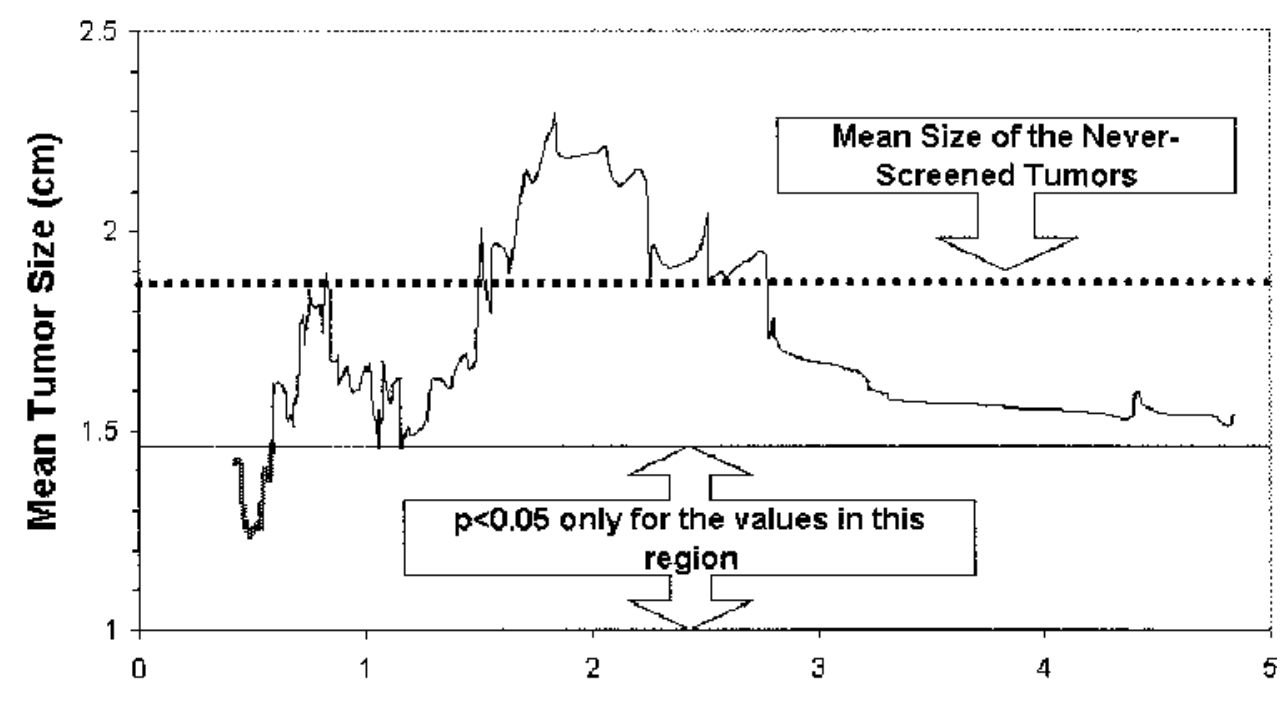

Figure 7. Mean sizes of the intervening cancers as a function of the time since the previous negative mammogram result as revealed by the 30 -data-point running average of the mean tumor size. See the legend to Table 1 for the method of calculation.

time since the previous negative mammogram

Table 2. Sizes of Subsequent-Screen-Detected Cancers Since the Previous Negative Mammogram

\begin{tabular}{lcccc}
\hline $\begin{array}{l}\text { Time Since } \\
\text { the Previous Negative } \\
\text { Mammogram (y) }\end{array}$ & $\mathrm{n}$ & $\begin{array}{l}\text { Mean } \\
\text { Diameter } \\
\text { (cm) }\end{array}$ & $\begin{array}{l}\text { Median } \\
\text { Diameter } \\
\text { (cm) }\end{array}$ & t-test \\
\hline $0.06-0.71$ & 23 & 0.88 & 1.00 & $\mathrm{p}=0.01$ \\
$0.02-0.93$ & 30 & 1.06 & 0.85 & $\mathrm{Ns}$ \\
$0.94-1.07$ & 30 & 1.25 & 1.00 & $\mathrm{Ns}$ \\
$1.07-1.11$ & 30 & 1.23 & 1.15 & $\mathrm{Ns}$ \\
$1.11-1.19$ & 30 & 1.29 & 1.10 & $\mathrm{Ns}$ \\
$1.19-1.29$ & 30 & 1.17 & 1.00 & $\mathrm{Ns}$ \\
$1.29-1.45$ & 30 & 1.09 & 0.90 & $\mathrm{Ns}$ \\
$1.45-1.81$ & 30 & 1.20 & 1.10 & $\mathrm{Ns}$ \\
$1.81-2.20$ & 30 & 1.14 & 1.05 & $\mathrm{Ns}$ \\
$2.23-3.49$ & 30 & 1.13 & 0.95 & $\mathrm{Ns}$ \\
$3.66-5.44$ & 30 & 1.12 & 1.20 & - \\
$5.44-10.42$ & 12 & 1.06 & 1.00 & - \\
$0.02-1.07$ & 60 & 1.15 & 1.00 & $\mathrm{Ns}$ \\
$0.02-1.11$ & 90 & 1.18 & 1.00 & $\mathrm{Ns}$ \\
$0.83-1.16$ & 72 & 1.20 & 1.00 & $\mathrm{Ns}$ \\
$1.83-2.16$ & 34 & 1.11 & 1.00 & $\mathrm{Ns}$ \\
$0.02-11.1$ & 312 & 1.17 & 1.00 & \\
\hline
\end{tabular}

The 312 subsequent-screen detected tumors were ordered from shortest to longest time since the previous negative mammogram, and all possible sequential groupings of 20 to 40 tumors were examined by Excel spreadsheet analysis. This yielded approximately 6,000 groups. Of these approximately 6,000 groups, only 9 groups of tumors, those containing tumors found within 0.7452 years of the previous negative exam, were significantly $(p<0.05)$ smaller than the mean size of the tumors found after this time; for no group containing tumors found after 0.7452 years was there any significant reduction in tumor size, compared with the tumors found later. Of these 9 groups, the one with the highest degree of statistical confidence $(p=0.012)$ was the group that contained 23 cancers found between 0.057 and 0.706 years after the previous negative exam; these 23 tumors were reduced in diameter by approximately $25 \%$, with a mean size of $8.8 \mathrm{~mm}$, in contrast to the mean size of $11.7 \mathrm{~mm}$ for the group of 312 subsequent-screen detected tumors as a whole, and $11.8 \mathrm{~mm}$ for the group of 289 subsequent-screen detected tumors found more than 0.706 years after the previous negative exam. The mean size of all of the tumors in the 9 groups of tumors that were significantly smaller because they were found within 0.7452 years the previous negative exam was reduced in diameter by approximately $25 \%$, with a mean size of 8.9 $\mathrm{mm}$, compared with the mean size of $11.7 \mathrm{~mm}$ for the group of 312 subsequent-screen detected tumors as a whole and $11.8 \mathrm{~mm}$ for the group of 387 subsequent-screen detected tumors found more than 0.7452 years after the previous negative exam. tial, ${ }^{21}$ and therefore the relationship between the number of cells in a tumor $(\mathrm{N})$ and time $(\mathrm{t})$ can be expressed as:

$$
\text { 1. } \mathbf{N}=\mathrm{N}_{\mathrm{O}} e^{\mathrm{rt}}
$$

where $N_{o}$ is the number of cells at time $t=0$ and $e$ is the exponential constant, $t$ is time, and $r$ is a constant, such that:

$$
\text { 2. } \mathrm{r}=\ln (2) / \mathrm{t}_{\mathrm{D}}
$$

where $t_{D}$ is the tumor doubling time.

Note that it is possible to estimate how long it should take for a tumor to grow from one size $\left(\mathrm{N}_{\mathrm{O}}\right)$ to another $(\mathrm{N})$ :

$$
\text { 3. } \mathrm{t}_{\mathrm{No}} \rightarrow_{\mathrm{N}}=\left(\ln \left(\mathrm{N} / \mathrm{N}_{0}\right)\right) / \mathrm{r}
$$

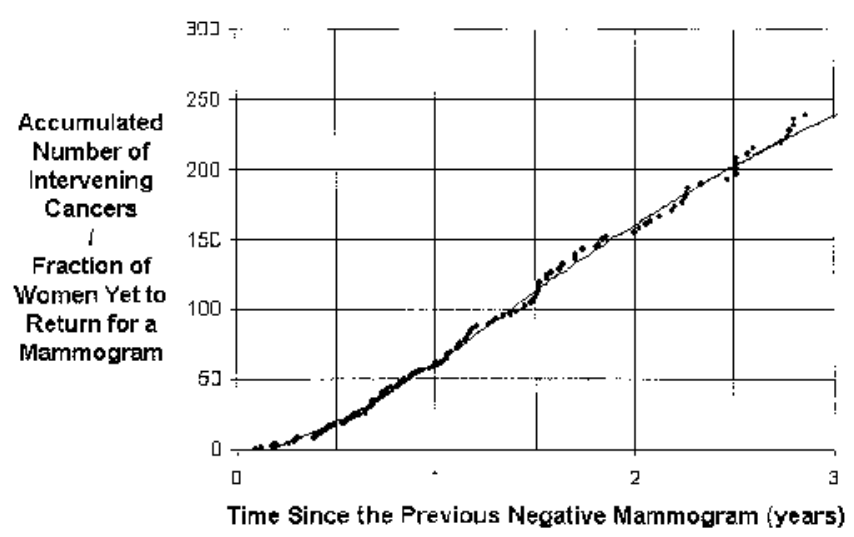

Figure 8. Accumulation of the nonmammographically detected cancers found in women with a previous negative mammogram result (intervening cancers). By dividing the number of these tumors by the fraction of women yet to undergo screening, the rate of appearance of these tumors could be visualized. Note that for approximately the first 6 months after the negative mammogram result, the rate of accumulation appears to be reduced. 


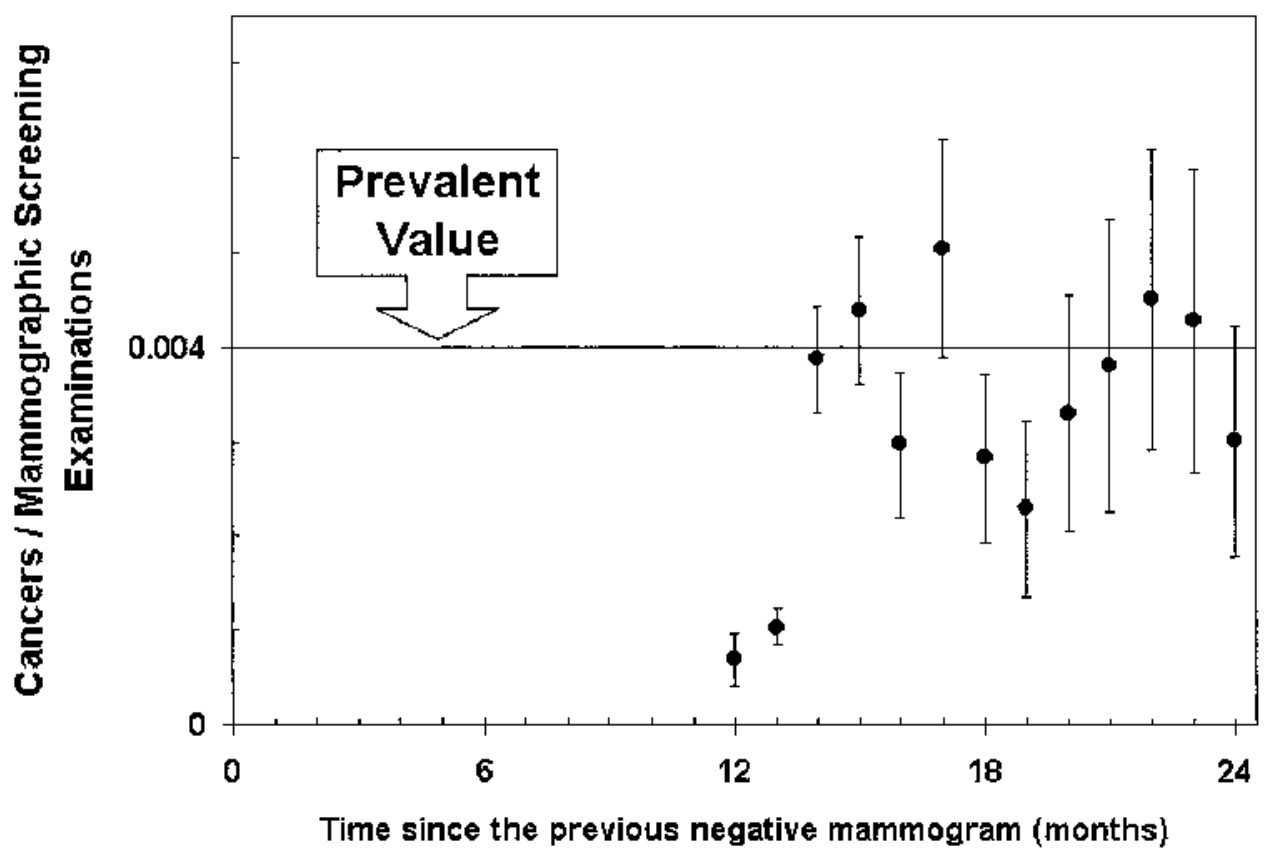

Figure 9. Mammographic screening yield as a function of the time since the previous negative mammogram result (in months). The estimate of the value of the screening yield of the prevalent cancers was be determined from the 16 invasive cancers found at screening among 4,096 women who had their first mammogram at $\mathrm{MGH}$ in 1998, generating an estimate of $3.90 \pm$ 0.98 invasive breast cancers per 1,000 mammographic examinations. 1998 was chosen because it allowed us to search the database for the previous 10 years to determine whether previous mammograms had been carried out.

In addition to characterizing invasive breast cancer by its growth rate, sometimes it is useful to describe tumor growth in terms of the sojourn time $\left(t_{S}\right)$, the time that it takes for tumors to grow from size $S_{m}$ (the size at which an individual invasive breast cancer will become operationally detectable by mammography) to size $S_{p}$ (the size at which an individual invasive breast cancer will become operationally detectable in the absence of mammography; see above). By convention, $S_{m}$ and $S_{p}$ will be referred to by tumor diameter. Let us call the number of cells in tumors of sizes $S_{m}$ and $S_{p}$ and $N_{m}$ and $N_{p}$. Therefore, for the simple case in which we approximate tumor detection with discrete values of $S_{m}$ and $S_{p}$ :

$$
\text { 4. } \mathrm{t}_{\mathrm{s}}=\left[\ln \left(\mathrm{N}_{\mathrm{p}} / \mathrm{N}_{\mathrm{m}}\right)\right] / \mathrm{r}
$$

Consider a set of A cancers, ranging in size from size $\mathrm{N}_{\mathrm{b}}$ (for biggest) to $\mathrm{N}_{\mathrm{s}}$ (for smallest), was found in a population of $Q$ women who underwent mammography for the first time at time $t=0 . Q \times I$ will be the number

Table 3. Various Estimates of the Breast Cancer Growth Rate

\begin{tabular}{|c|c|}
\hline Reference & Tumor Growth Rate Estimate \\
\hline This paper & Median doubling time $=130$ days \\
\hline Fournier et $\mathrm{al}^{3}$ & Geometric mean doubling time $=225$ days \\
\hline Lundgren $^{4}$ & Mean doubling time $=211$ days \\
\hline Spratt et al ${ }^{56}$ & Median doubling time $=260$ days \\
\hline Peer et $\mathrm{al}^{8}$ & $\begin{array}{l}\text { Geometric mean doubling time }=80 \text { days (women, } \\
\text { age }<50 \text { ) }\end{array}$ \\
\hline Peer et $a l^{8}$ & $\begin{array}{l}\text { Geometric mean doubling time }=157 \text { days (women, } \\
\text { age } 50-70 \text { ) }\end{array}$ \\
\hline Peer et $\mathrm{al}^{8}$ & $\begin{array}{l}\text { Geometric mean doubling time }=188 \text { days women, } \\
\text { age }>70)\end{array}$ \\
\hline Kuroishi et $\mathrm{al}^{7}$ & Geometric mean doubling time $=174$ days \\
\hline
\end{tabular}

of cancers that arise in this population of women per year, where I is the invasive breast cancer incidence for this population of $\mathrm{Q}$ women, I being calculable from standard sources, such as the SEER national database. Let $-t_{b}$ be the time when the largest tumor in the set of tumors (which is size $\mathrm{Nb}$ at time $\mathrm{t}=0$ ) was size $\mathrm{N}_{\mathrm{s}}$. We can calculate the value of $t_{b}$ with equation 3 :

$$
\text { 5. } \mathrm{t}_{\mathrm{b}}=\left(\ln \left(\mathrm{N}_{\mathrm{b}} / \mathrm{N}_{\mathrm{s}}\right)\right) / \mathrm{r}
$$

Let us consider the case in which we have chosen a value of $N_{s}$ so that it is sufficiently small that no cancers smaller than $\mathrm{N}_{\mathrm{s}}$ are detected. Let us define $\mathrm{Z}$ as the number of cancers that have grown to size $\mathrm{N}_{\mathrm{s}}$ per unit of time. Z, in terms of Q and I, is determined as follows:

$$
\text { 6. } Z=(Q \times I)
$$

where $\mathrm{Z}$, in terms of the A cancers between sizes $\mathrm{N}_{\mathrm{b}}$ and $\mathrm{N}_{\mathrm{s}}$ seen by mammography at time $\mathrm{t}=0$ is:

$$
\text { 7. } \mathrm{Z}=\mathrm{A} / \mathrm{t}_{\mathrm{b}}
$$

\begin{tabular}{|c|c|c|}
\hline Reference & Study Population & $\begin{array}{l}\text { Mean Sojourn } \\
\text { Time (y) }\end{array}$ \\
\hline This paper & $\mathrm{MGH}$ & 1.7 \\
\hline Shapiro et al ${ }^{9}$ & HIP & 1.3 \\
\hline Hutchison and Shapiro ${ }^{10}$ & HIP & 1.7 \\
\hline Walter and Day ${ }^{14}$ & HIP & 1.7 \\
\hline Albert et al ${ }^{11}$ & HIP & 1.8 \\
\hline Tallis and Sarafaty ${ }^{13}$ & Melbourn & 1.8 \\
\hline Zelen and Feinleib ${ }^{12}$ & HIP & 2.4 \\
\hline Duffy et al ${ }^{15}$ & Swedish, age $70-74$ & 3.8 \\
\hline Chen et $\mathrm{al}^{16,17}$ & Swedish, age $40-49$ & 2.4 \\
\hline Chen et $a^{16,17}$ & Swedish, age $50-59$ & 3.7 \\
\hline Chen et $\mathrm{al}^{16,17}$ & Swedish, age 60-69 & 4.2 \\
\hline
\end{tabular}

Table 4. Various Estimates of the Breast Cancer Sojourn Time 
Therefore, combining equations 6 and 7 yields:

$$
\text { 8. } t_{b}=A / Q \times I
$$

and then combining equations 5 and 8 yields:

$$
\text { 9. }\left[\left(\ln \left(\mathrm{N}_{\mathrm{b}} / \mathrm{N}_{\mathrm{s}}\right)\right) / \mathrm{r}\right]=\mathrm{A} / \mathrm{Q} \times \mathrm{I}
$$

Rearranging yields:

$$
\text { 10. } r=1 /\left[\mathrm{A} / \mathrm{Q} \times \mathrm{I} \times\left(\ln \left(\mathrm{N}_{\mathrm{b}} / \mathrm{N}_{\mathrm{s}}\right)\right)\right]
$$

Because the tumor doubling time, $t_{D}$, is inversely related to $r$ (equation 2):

$$
\text { 11. } \mathrm{t}_{\mathrm{D}}=[\ln (2) \mathrm{A}] /\left[\mathrm{Q} \times \mathrm{I} \times\left(\ln \left(\mathrm{N}_{\mathrm{b}} / \mathrm{N}_{\mathrm{s}}\right)\right)\right]
$$

Note also that if we represent the tumor size by diameter $\left(\mathrm{S}_{\mathrm{b}}\right.$ and $\left.\mathrm{S}_{\mathrm{s}}\right)$ rather than cell number $\left(\mathrm{N}_{\mathrm{b}}\right.$ and $\left.\mathrm{N}_{\mathrm{s}}\right)$ :

$$
\text { 12. } \mathrm{t}_{\mathrm{D}}=[\ln (2) \mathrm{A}] /\left[\mathrm{Q} \times \mathrm{I} \times 3\left(\ln \left(\mathrm{S}_{\mathrm{b}} / \mathrm{S}_{\mathrm{s}}\right)\right)\right]
$$

To this point, we have considered the case in which all tumors have the same doubling time, $t_{D}$, and the same growth rate constant, $r$, rather than a distribution of these values. However, it can be shown mathematically that equation 12 will yield exactly the same results if tumor doubling times are distributed symmetrically (e.g., normally distributed) around a mean doubling time, $t_{\mathrm{D}}$. For a symmetric distribution, the mean doubling time will equal the median doubling time; however, if the data are skewed, the mean and median may differ slightly. Therefore, assuming a symmetric distribution, the median doubling time $\left(\mathrm{t}_{\mathrm{Dm}}\right)$ will be:

$$
\text { 13. } \mathrm{t}_{\mathrm{Dm}}=[\ln (2) \mathrm{A}] /\left[\mathrm{Q} \times \mathrm{I} \times 3\left(\ln \left(\mathrm{S}_{\mathrm{b}} / \mathrm{S}_{\mathrm{s}}\right)\right)\right]
$$

Let us call $\mathrm{Fp}_{\mathrm{Sb}-\mathrm{Ss}}$ the fraction of cancers with diameters larger than $S_{s}$ but smaller than size $S_{b}$ that are expected to be detected by palpation before they would be seen by mammography, and $\mathrm{Fm}_{\mathrm{Sb}-\mathrm{Ss}}$ the fraction of cancers with diameters larger than $S_{s}$ but smaller than size $S_{b}$ that are expected to be missed by mammography. The value of $\mathrm{Fp}_{\mathrm{Sb}-\mathrm{Ss}}$ can be estimated from the cumulative distribution of the sizes of tumors seen in women who have never undergone screening (never-screened cancers), as noted earlier. The value of $\mathrm{Fm}_{\mathrm{Sb}-\mathrm{Ss}}$ can be estimated because the tumors missed at screening will appear later, either as mammographically detected cancers found on subsequent examinations or as nonmammographically detected cancers (intervening cancers and subsequent-screen-detected cancers). It is then a matter of estimating the size each of these tumors would have been at the time of screening. This calculation requires an estimate of the tumor doubling time, which is also the object of the overall calculation of the doubling time; however, the data are compatible with only a single solution to both variables, which can be arrived at by treating them as the products of two linked nonlinear equations requiring simultaneous solution, which was accomplished by automating the solution in Microsoft Excel (Figure 2). With use of all this information, it is now possible to estimate the median doubling time $\left(\mathrm{t}_{\mathrm{Dm}}\right)$ as:

$$
\text { 14. } \begin{aligned}
\mathrm{t}_{\mathrm{Dm}}= & {\left[\ln (2) \times \mathrm{a} \times\left(1 /\left(1-\mathrm{Fm}_{\mathrm{Sb}-\mathrm{Ss}}\right)\right) \times\right.} \\
& \left.\left(1 /\left(1-\mathrm{Fp}_{\mathrm{Sb}-\mathrm{Ss}}\right)\right)\right] /\left[\mathrm{Q} \times \mathrm{I} \times 3\left(\ln \left(\mathrm{S}_{\mathrm{b}} / \mathrm{S}_{\mathrm{s}}\right)\right)\right]
\end{aligned}
$$

\section{References}

1. Michaelson J, Halpern E, Kopans D: A computer simulation method for estimating the optimal intervals for breast cancer screening. Radiology 1999;212:551-560.

2. Michaelson J: The role of molecular discreteness in normal and cancerous growth. Anticancer Res 1999;19(6A):4853-4867.

3. Fournier DV, Weber E, Hoefken W, et al: Growth rate of 147 mammary carcinoma. Cancer 1980;45:2198-2207.

4. Lundgren B: Observations on the growth rate of breast carcinomas and its possible implications for lead time. Cancer 1977;40:1722-1725.

5. Spratt JA, Von Fournier D, Spratt JS, et al: Mammographic assessment of human breast cancer growth and duration. Cancer 1993;71:2020-2026.

6. Spratt JS, Spratt T: Rates of growth of pulmonary metastases and survival. Ann Surg. 1964;159:161-171.

7. Kuroishi T, Tominaga S, Morimoto T, et al: Tumor growth rate and prognosis of breast cancer mainly detected by mass screening. Jpn J Cancer Res 1990;81:454-462.

8. Peer PGM, van Dick JAAM, Hendricks JHCL, et al: Age-dependent growth of primary breast cancer. Cancer 1993;71:3547-3551.

9. Shapiro S, Goldberg, JD, Hitchison GB: Lead time in breast cancer detection and implications for periodicity of screening. Am J Epidemiol 1974;100: 357-266.

10. Hutchison GB, Shapiro S: Lead time gained by diagnostic screening for breast cancer. J Natl Cancer Inst 1968;41:665-681.

11. Albert A, Gertman PM, Louis TA, et al: Screening for the early detection of cancer II The impact of screening on the natural history of the disease Math Biosci 1978;10:61-109.

12. Zelen M, Feinleib M: Optimal scheduling of examinations for the early detection of disease Biometika 1969;56:601-614.

13. Tallis GM, Sarafaty G: On the distribution of the time to reporting cancers with application to breast cancer in women. Math Biosci 1974;128:371376.

14. Walter SD, Day NE: Estimation of the duration of a preclinical disease state using screening data. Am J Epidemiol 1983;118:865-886.

15. Duffy SW, Day NE, Tabar L, et al: Markov models of breast tumor progression: some age-specific results. J Natl Cancer Inst Monogr 1997;22:9397.

16. Chen HH, Duffy SW, Tabar L, et al: Markov chain models for progression of breast cancer. Part I: tumor attributes and the preclinical screendetectable phase. J Epidemiol Biostatistics 1997;2:9-23.

17. Chen HH, Duffy SW, Tabar L, et al: Markov chain models for progression of breast cancer. Part II: prediction of outcomes from different screening regimes. J Epidemiol Biostatistics 1997;2:25-35.

18. Michaelson JS, Satija S, Moore R, et al: Observations on invasive breast cancers diagnosed in a service screening and diagnostic breast imaging program. J Womens Imaging 2001;3:99-104.

19. Michaelson JS, Satija S, Moore R, et al: Estimates of the sizes at which breast cancers become detectable on mammographic and clinical grounds. J Womens Imaging 2003;5:3-10.

20. Norton L: A Gompertzian model of human breast cancer growth. Cancer Res. 1988;48:7067-7071.

21. Surbone A, Norton L: Kinetic concepts in the treatment of breast cancer. Ann N Y Acad Sci 1993;698:48-62.

22. Hunt KA, Rosen EL, Sickles EA: Outcome analysis for women undergoing annual versus biennial screening mammography: a review of 24,211 examinations. AJR Am J Roentgenol 1999;173:285-289.

23. Tabar L, Dean PB, Duffy SW, et al: A new era in the diagnosis of breast cancer. Surg Oncol Clin N Am 2000;9:233-277.

24. Tubiana M, Koscielny S: The natural history of human breast cancer: implications for a screening strategy. Int J Radiat Oncol Biol Phys 1990;19: 11171120 . 
25. Koscielny S, Tubiana M, Le MG, et al: Breast cancer: relationship between the size of the primary tumor and the probability of metastatic dissemination. Br J Cancer 1984;49:709-715.

26. Kopans DB: Breast Imaging. Second Edition. Philadelphia: LippincottRaven, 1997.

27. Michaelson JS, Kopans DB, Cady B: The breast cancer screening interval is important. Cancer 2000;88:1282-1284.

28. Hunt KA, Rosen EL, Sickles EA: Outcome analysis for women undergoing annual versus biennial screening mammography: a review of 24,211 examinations. AJR Am J Roentgenol 1999;173:285-289.

29. Michaelson JS: Using information on breast cancer growth, spread, and detectability to find the best ways to use screening to reduce breast cancer death. J Womens Imaging 2001;3:54-57.
Address correspondence to:

James S. Michaelson, PhD Division of Surgical Oncology

Cox Building, Room 626

Massachusetts General Hospital

100 Blossom Street

Boston, MA 02114

E-mail: michaelj@helix.mgh.harvard.edu 